\title{
Locating temporary shelter areas after an earthquake: A case for Turkey
}

\author{
Firat Kılc1 ${ }^{a}$, Bahar Yetiş Kara ${ }^{\mathrm{a}, *}$, Burçin Bozkaya ${ }^{\mathrm{b}}$ \\ a Department of Industrial Engineering, Bilkent University, 06800, Ankara, Turkey \\ ${ }^{\mathrm{b}}$ School of Management, Sabanci University, 34956, Istanbul, Turkey
}

\section{A R T I C L E I N F O}

Article history:

Received 26 October 2013

Accepted 24 November 2014

Available online 9 December 2014

\section{Keywords:}

Location

Shelter site selection

Humanitarian logistics

Practice of OR

Disaster planning

\begin{abstract}
A B S T R A C T
In this study, we propose a mixed integer linear programming based methodology for selecting the location of temporary shelter sites. The mathematical model maximizes the minimum weight of open shelter areas while deciding on the location of shelter areas, the assigned population points to each open shelter area and controls the utilization of open shelter areas. We validate the mathematical model by generating a base case scenario using real data for Kartal, Istanbul, Turkey. Also, we perform a sensitivity analysis on the parameters of the mentioned mathematical model and discuss our findings. Lastly, we perform a case study using the data from the 2011 Van earthquake.
\end{abstract}

(C) 2014 Elsevier B.V. All rights reserved.

\section{Introduction}

The International Federation of Red Cross and Red Crescent Societies (IFRC) defines a disaster as "a sudden, calamitous event that seriously disrupts the functioning of a community or society and causes human, material, and economic or environmental losses that exceed the community's or society's ability to cope using its own resources" (IFRC, "What is a disaster?").

Turkey is among the countries that are especially vulnerable to natural disasters. Throughout history, many disasters have occurred in the geography where Turkey is located. According to Özmen, Nurlu, Kuterdem, and Temiz (2005), 650,654 households have been destroyed by disasters since 1900 . Total destruction in Turkey from these disasters is broken down in Table 1 below.

Turkey is not subject to tornados or hurricanes, but earthquakes, landslides, floods, rock falls and avalanches frequently occur. The latter four disaster types are usually small-scaled, with relatively little or no death toll. Earthquakes, however, are the most feared type of disaster in Turkey, as many lives are often lost. Several fault lines run through Turkey, but the North Anatolian Fault, from Thrace to Northeast Turkey, is the most active one. The danger posed by this fault line is evident when one compares the percentages of surface area and population to be affected in a high-magnitude earthquake.

There are five different earthquake zones in Turkey, with Table 2 showing them ordered according to degrees, with the first being the most dangerous and the fifth the least dangerous. According

\footnotetext{
* Corresponding author. Tel.: +90 312 2901262; fax: +90 3122664054

E-mail address: bkara@bilkent.edu.tr (B.Y. Kara).
}

to Özmen, Nurlu, and Güler (1997), 44 percent of Turkey's population lives in the first degree zones.

Since the beginning of the twentieth century, approximately 110 destructive earthquakes have occurred in Turkey. About 15 of those had death tolls greater than 1000 . The most important ones in terms of causalities are the 1939 Erzincan earthquake and the 1999 Marmara earthquake.

The Erzincan earthquake was the most devastating earthquake in Turkey's history. It had a magnitude of 7.8 on the Richter scale and left 33,000 people dead and hundreds of thousands homeless. The 1999 Marmara earthquake had a magnitude of 7.6 on the Richter scale and killed about 17,000 people, injured nearly 50,000 people and left about 500,000 homeless. The aftershocks of this earthquake lasted several months with the greatest aftershock in Düzce, with a magnitude of 7.2. That event killed about 1000 people, while leaving thousands of homes damaged and thousands of people homeless ( $\mathrm{Al}$ Jazeera Turk, 2013). The recorded financial damage of the Marmara earthquake was about 3-6.5 billion US dollars (Aslanzadeh, Rostami, \& Kardar, 2009).

On 16 March 2000, seven months after the 1999 Kocaeli Earthquake, the Turkish daily Radikal published an article with data on the number of residents in temporary shelter areas. According to this article, in Kocaeli, Sakarya, Yalova, Bolu and Düzce, around 91,000 people were still living in tents. The number of people living in the shelter areas established in each city and their utilization are given in Table 3a.

The Turkish daily Milliyet published a series of articles between 11 and 16 August 2000 reporting the numbers of people that were still homeless and living in shelters exactly a year after the disaster. The number of people living in the tents had decreased since Radikal's 
Table 1

Types of disasters and their respective damages since 1900 (Özmen et al., 2005).

\begin{tabular}{lcc}
\hline Type of disaster & $\begin{array}{l}\text { Number of households } \\
\text { destroyed }\end{array}$ & Percentage \\
\hline Earthquake & 495,000 & 79 \\
Landslide & 63,000 & 10 \\
Flood & 61,000 & 9 \\
Rockfall & 26,500 & 4 \\
Avalanche & 5,154 & 1 \\
Total & 650,654 & 100 \\
\hline
\end{tabular}

article, but the Milliyet article reported that although pre-fabricated houses had been constructed offering better living conditions than the tents, people continued living in the tents because the new housing areas were located very far away from city centers. The number of people living in tents and pre-fabricated houses can be found in Table 3b.

The aim of this study is to improve the disaster preparedness of Turkey by developing a methodology for selecting shelter site locations. In this section, we provide brief information about disasters and past data related to shelter areas in Turkey. In Section 2, we present the principles and standards used while constructing the shelter areas and discuss the Turkish Red Crescent's methodology on shelter site selection. In Section 3, we briefly summarize the related literature. In Section 4, we define the problem and propose a mathematical model to address the problem. In Section 5, we discuss the computational studies that we performed using the mathematical model, and in Section 6, we conclude this article by briefly summarizing the study and pinpointing possible future research areas.

\section{Current methodology on shelter site selection in Turkey}

After a large-scale disaster, houses become damaged or destroyed, and a notable number of residents become homeless. Because people need to continue their everyday life, they must reside in a temporary place until the disaster recovery process is completed. Because of this, to address the needs of the affected population, shelter areas are established. Ideally, these areas should be designed with respect to quality measurements.

In 1997, several humanitarian organizations and the International Red Crescent and Red Cross Movement initiated a project to improve the quality of post-disaster humanitarian operations (Sphere Project 2011). The philosophy is based on two principles: i) the affected population has the right to live with dignity and receive necessary assistance, and ii) whenever human suffering is caused by disaster or such conflict, any necessary action should be taken in order to suppress it. The respective project that defines these quality measurements is called "The Sphere Project". This publication is an important source of information in the humanitarian sector as it is the most comprehensive document that defines the standards of humanitarian relief operations, compiled by the most experienced organizations in the sector.
Table 3a

Utilization and population of shelter areas in five cities in March 2000 (Radikal, 2000).

\begin{tabular}{lcc}
\hline City & Number of people & Utilization (percent) \\
\hline Kocaeli & 18500 & 100 \\
Sakarya & 906 & 20 \\
Yalova & 2547 & 74 \\
Bolu & 16648 & 100 \\
Düzce & 53000 & 90 \\
\hline
\end{tabular}

Table 3b

The number of people living in temporary shelters in five cities in August 2000 (Milliyet, 2000).

\begin{tabular}{lrl}
\hline City & $\begin{array}{c}\text { Number } \\
\text { in tents }\end{array}$ & $\begin{array}{l}\text { Number in } \\
\text { pre-fabricated houses }\end{array}$ \\
\hline Kocaeli & 9,865 & 55,399 \\
Sakarya & 229 & 38,131 \\
Yalova & 0 & 15,946 \\
Bolu & 10,591 & 14,296 \\
Düzce & 8,232 & 22,822 \\
\hline
\end{tabular}

Because of shelter areas' importance, they must be strategically planned. Ensuring sufficient relief materials such as tents, shelter kits, and construction kits is of course necessary. The responsible organization should also ensure that established shelter areas are located within a distance from threat zones, while considering the need for and distance of safe routes between the shelter area and the homes of affected people and from the shelter area to essential service facilities. Also, ownership and usage rights of each shelter area should be pre-determined and any necessary permission should be obtained.

In Turkey, the Red Crescent is the main body responsible for establishing temporary shelter areas. After a disaster, it determines the shelter locations and supplies the necessary amount of tents in order to provide residence to the homeless. It is also responsible for supplying enough food and non-food items for those living in the shelter areas and for ensuring the security of the shelters.

Especially in disaster prone areas like Istanbul, the Turkish Red Crescent defines the eligible sites for shelter areas before the disaster. In mid 2000s, experts stated that an earthquake is anticipated in Istanbul within 10 years. Because of this, the Turkish Red Crescent and Istanbul Greater Municipality conducted a study in order to define the potential location of temporary shelter areas. The Turkish Red Crescent has defined 10 criteria to rank potential shelter areas that can be used in any part of Turkey, which are listed below.

- Transportation of relief items: this criterion measures the accessibility of the shelter area. If main roads are closer to the shelter areas, transportation of relief items becomes easier.

- Procurement of relief items: relief items are purchased from a market, supermarket, or warehouse. The closer such an establishment is to the shelter area, the less costly it will be to procure the items.

Table 2

Surface area and population with respect to five earthquake zones in Turkey (Özmen et al., 1997).

\begin{tabular}{|c|c|c|c|c|c|c|}
\hline $\begin{array}{l}\text { Earthquake } \\
\text { zones }\end{array}$ & $\begin{array}{l}\text { Surface } \\
\text { area square kilometers }\end{array}$ & Percentage & $\begin{array}{l}\text { Population } \\
\text { (1990) }\end{array}$ & Percentage & $\begin{array}{l}\text { Forecasted } \\
\text { population (1997) }\end{array}$ & Percentage \\
\hline First degree & 328,995 & 42 & $25,052,683$ & 44 & $28,498,740$ & 45 \\
\hline Second degree & 186,411 & 24 & $14,642,950$ & 26 & $16,674,656$ & 26 \\
\hline Third degree & 139,594 & 18 & $8,257,582$ & 15 & $9,334,138$ & 15 \\
\hline Fourth degree & 97,894 & 12 & $7,534,083$ & 13 & $8,129,711$ & 13 \\
\hline Fifth degree & 32,051 & 4 & 985,737 & 2 & $1,107,757$ & 2 \\
\hline Total & 784,945 & 100 & $56,473,035$ & 100 & $63,745,002$ & 100 \\
\hline
\end{tabular}


- Healthcare institutions: it is favorable if a shelter area is close to a functioning hospital and/or medical clinic(s) so that care can be provided to those who need it.

- Topography of the terrain: this criterion measures the suitability of the candidate location for building and daily life. Piedmont, valley, savannah and stream beds are several examples of possible topographies. Among these examples, savannah is the most desirable as building and living on a plain is easier than in a hilly area.

- Type of terrain: this criterion measures the hardness of the soil. Hard soil is less affected by rain and thus is easier to build and live on.

- Slope of the terrain: this criterion measures the slope of the terrain upon which the shelter area is established. According to the Turkish Red Crescent, the slope of the terrain of a candidate location cannot exceed 7 percent and it is better if the slope is between 2 percent and 4 percent.

- Electrical infrastructure: electricity is important for residents to pursue their daily lives. Many devices used in daily life, including heat sources, run on electricity. Shelter areas should have electrical infrastructure where possible.

- Sanitary system: water is one of the most important human needs. In addition to sustaining life, it is used for cooking, cleaning, and personal hygiene. To ensure health, water must be properly treated and disposed of. Because of this, sewage infrastructure is important for a shelter area.

- Flora of the terrain: trees provide oxygen and shade, which is useful during hot weather. Because of this, dense flora incorporating trees is preferable for shelter areas.

- Ownership: it is easier to get construction permission if the shelter area is publicly owned than if privately owned.

It is notable that although it seems imminent, cost is not an issue here. Locating shelter areas after a disaster is a humanitarian act and in such cases, quality of service surely outperforms the importance of cost. Thus, the Turkish Red Crescent is willing to install enough shelter sites to provide adequate service regardless of its cost.

These 10 criteria are not equally weighed. They all have respective weights and each candidate shelter location receives a point between 0 and 1 for each criterion, which are determined by the Turkish Red Crescent officials beforehand and assigned to each candidate location by inspecting it. The grade point of a potential shelter area is the convex combination of the points obtained from these 10 criteria. The Turkish Red Crescent sorts the potential shelter areas with respect to their grade points and in a disaster, starts construction in the ones with the highest grade points until enough shelter areas are functioning to house all the affected people.

Careful observation of the methodology of the Turkish Red Crescent in choosing shelter sites reveals that the methodology could be improved. The organization does not consider the distance between people's actual homes and the shelter areas. This may result in a situation where a certain district is very far from all open shelters. This will make it hard to reach any open shelter areas from that district, which is not favorable.

Utilization of shelter areas is also important. As evident from Table 3a, the shelter area utilizations differ significantly. If there is no balance between shelter areas, one shelter area may be full while others are half utilized. This situation is not desirable as it is more convenient to live in less utilized shelter areas. To overcome this issue, the utilization of each area and the pair-wise utilization difference of the areas should be considered while selecting the shelter area locations and assigning population to them.

In this study, a mixed integer linear programming formulation, which chooses the location of shelter areas, controls their utilization and assigns population to operating shelter areas, is formulated.

\section{Literature review}

Many studies in the literature discuss facility location in disaster relief. These studies can be categorized into three problems: the emergency medical center location, the relief material warehouse location and the shelter site location problem. Although these three types of facilities have different usages, these problems have similar objectives such as maximizing the total number of people covered, minimizing the distance between facilities and the affected population and selecting the most reliable set of facilities. Thus, in this section, we discuss notable studies regarding these problems.

\subsection{Emergency medical center location problem}

This problem addresses the location of emergency medical centers that are established in order to provide medical attention after an emergency due to a disaster.

Dekle, Lavieri, Martin, Emir-Farinas, and Francis (2005) and Ablanedo-Rosas, Gao, Alidaee, and Teng (2009) address the emergency medical center location problem using set-covering models. Jia, Ordóñez, and Dessouky (2007a) consider the same problem by introducing randomness using a scenario analysis, however they face computational limitations. These limitations are resolved by Lu et al. (2009)'s heuristic based on an ant colony algorithm. Apart from these deterministic models, Verma and Gaukler (2011) define a two-stage stochastic programming model to determine the location of the facilities while taking transportation of the items into account. While selecting the locations of emergency medical centers, Gül(2008) combines existing facilities with temporary ones and perform a case study based on Istanbul, Turkey. Huang, Kim, and Menezes (2010) design a variation of the p-median model with the assumption that a center at a node may fail to respond. Wang and Zang (2006) take emergency occurrence probability for a specific region into account. Paul and Batta (2008) and Chang, Tseng, and Chen (2007) optimize locations of the facilities and crew allocation simultaneously. Toro-Díaz, Mayorga, Chanta, and McLay (2013) deals with ambulance location and dispatching decision and provides a genetic algorithm based optimization scheme. Lu (2013) offers a p-center based method for this problem by minimizing the maximum response time and introducing randomness by using robust optimization methods.

\subsection{Relief material warehouse location problem}

The relief material warehouse location problem addresses the decision process of locating storage facilities for relief items such as canned foods, tents, blankets and water.

Balcik and Beamon (2008) deal with the prepositioning of relief supplies by formulating a variant of the maximum coverage location problem (MCLP) and Gunneç (2007) considers a similar problem using a variant of the uncapacitated facility location problem (UCFL). Hale and Moberg (2005) formulate a deterministic set-covering problem and propose a four-step site decision process. Similarly, Murali, Ordóñez, and Dessouky (2012) deal with this problem using a variation of the maximum covering location problem (MCLP). Duran, Gutierrez, and Keskinocak (2011) consider the prepositioning problem for CARE International. For the same problem, Görmez, Köksalan, and Salman (2011) provide a two-stage multi-objective model that maximizes the total number of refugees covered and decides on the location of facilities. Yushimito et al. (2012) and Li, Jin, and Zhang (2011) provide a heuristic algorithm for selecting prepositioning areas that tries to find a pre-specified number of facilities to cover all the demand points and minimize urgency. Han et al. (2011) perform a location-allocation study and their model optimally locates facilities and allocates disaster areas to opened facilities while minimizing total travel time. Campbell and Jones (2011) and Jia, Ordóñez, and Dessouky (2007b) consider a stochastic prepositioning approach with a single 
demand point, where the demand depends on a probability distribution. Rawls and Turnquist (2010) and Mete and Zabinsky (2010) provide a stochastic optimization problem to determine the location and quantity of emergency supplies. Rath and Gutjahr (2014) provide a heuristic method for determining the location of the warehouses and routing of the relief items. Apart from the mathematical studies, Kapucu, Lawther, and Pattison (2007) provide insight on determining potential sites before selecting final sites.

\subsection{Shelter site location problem}

Shelter areas are established for the affected people who lost their homes after a disaster. The shelter site location problem is used for determining the locations of such areas.

Anping (2010) considers the shelter site location problem after a disaster by formulating two deterministic mathematical models that are variations of the maximum set-covering problem. Li and Jin (2010) consider the stochastic nature of hurricanes and introduced this randomness by generating different scenarios and respective occurrence probabilities. Dalal et al. (2007) consider the same problem, using a heuristic based clustering approach and Liu et al. (2011) define the criteria for the shelter location problem and their ideas may be used while identifying potential locations. Li et al. (2012) propose a bi-level optimization method for selecting the location of shelter sites after a hurricane and provide a study with data based on North Carolina. Alçada-Almeida, Tralhão, Santos, and CoutinhoRodrigues (2009) locate shelter sites and identify evacuation routes by using a multi-objective approach. Coutinho-Rodrigues, Tralhão, and Alçada-Almeida (2012) provide a solution method for a multiobjective shelter site location and routing problem.

Anping (2010) and Li and Jin (2010)'s studies are based on cover models. Dalal et al. (2007) present a clustering based approach that assumes there will be enough space to build a shelter area in each cluster. However, this assumption may not always hold. Because of this, making a decision from a pre-determined set of potential shelter areas and their capacities is a more solid approach.

To the best of our knowledge, there is no study that determines shelter area locations from a set of candidates and assigns populations to those areas while trying to keep all shelter areas as utilized as possible and minimizing their pairwise utilization difference. Our study considers these factors, thus offering an important contribution to the literature.

Moreover, apart from obtaining a solution in case of an emergency, decision makers will be able to obtain solutions for a set of scenarios using the model that is explained in the next section. Thus, our study offers improvements to the methods used in the planning stage of the disaster cycle, which is quite important in disaster relief.

\section{Problem definition and proposed model}

As noted, the Turkish Red Crescent ranks potential shelter areas with respect to a weight function composed of 10 criteria, and opens areas until there is enough space to house all the affected population. However, the Turkish Red Crescent does not consider distances between districts and shelter areas, nor does it consider shelter area utilizations.

In this study, we improved the Turkish Red Crescent's current methodology on the subject by considering their 10 criteria, determining district-shelter area assignments, and controlling the utilization of open shelter areas and proposed a mixed integer linear programming model. In this case, a candidate shelter area is said to be "open" if it is established and there exists population assigned to it. On the other hand, a candidate shelter area is said to be "closed' if sheltering facilities are not established on this candidate location premises.
To maintain the Turkish Red Crescent's methodology as much as possible, a similar weight function is devised for candidate shelter areas. However, as the number of criteria increases in this weight function, the reliability of the function decreases because of the interaction between different criteria. Thus, we omitted the criteria related to procurement and transportation of the relief items from the weight function as they are directly measurable using the travel distance between shelter areas and the nearest main road. Similarly, the criterion for health institutions is omitted from the weight function as it is sufficient to calculate the distance between shelter areas and hospitals. We furthermore assume that the remaining seven of the 10 criteria, namely, structure, slope, type and flora of the terrain, existence of electrical and sanitary systems and ownership are included in the weight function because they are not directly measurable.

In practice, capacities of health institutions are also important. However, the Turkish Red Crescent does not take the capacities of such institutions into account. Thus, we do not include the capacity of the health institutions in the model. If necessary, this issue can be addressed by limiting the capacity of the shelter areas to the total capacity of nearby hospitals.

For a settlement to be spacious, at least 3.5 square meters covered living space should be assigned to each person in the shelter area. Also, there should be at least 45 square meters space assigned for utilities such as roads, sanitation, health, education and nutrition if these services are to be provided within the shelter area. Thus, we include these measures while calculating the used capacity for each candidate location.

In order to feel safe after a disaster, a person would want to be with his/her acquaintances. Also, being close to acquaintances is very important to maintain the social structure of the society. We assumed that a person has a degree of familiarity with the people that live in the same district with him/her. Moreover, all affected people would prefer to be located at the closest possible shelter area. Thus, we assumed that there is no population splitting (i.e. all people that live in the same district are assigned to one single location, which is the closest one to them).

The objective of the mathematical model is to select the best possible combination of shelter areas (i.e. maximize the minimum weight of open shelter areas) while assigning each district to the closest open shelter area, obeying the capacity of shelter areas, controlling the minimum utilization and pair-wise utilization difference of open shelter areas, and making sure that each open shelter area has a main road connection and a health institution within a certain threshold distance.

The sets, parameters, and the model formulation are presented below.

\section{Sets}

I: set of candidate locations

$J$ : set of districts

\section{Parameters}

$w_{i}$ : weight of candidate location $i$, between 0 and 1

$d_{i}^{\text {health }}$ : distance between candidate location $i$ and nearest health center

$d_{i}^{\text {road }}$ : distance between candidate location $i$ and nearest main road Demand $_{j}$ : total demand of district $j$ in terms of square meters cap $_{i}$ : capacity of candidate location $i$

distSorted $_{i j}$ : $i$ th closest candidate location index to demand point $j$ DistHealth: threshold value for shelter area-health center distance DistRoad: threshold value for shelter area-main road distance $\alpha$ : threshold value for pair wise utilization difference of candidate shelter areas

$\beta$ : threshold value for minimum utilization of open shelter areas 


\section{Decision variables}

$$
\begin{aligned}
& x_{i}:\left\{\begin{array}{r}
1 \text { if candidate location } i \text { is chosen as a shelter area } \\
0 \text { otherwise }
\end{array}\right. \\
& y_{i j}:\left\{\begin{array}{r}
1 \text { if district } j \text { is assigned to location } i \\
0 \text { otherwise }
\end{array}\right. \\
& w_{\text {min }} \text { : minimum weight of operating candidate shelter areas }
\end{aligned}
$$

\section{Formulation}

$$
\begin{aligned}
& \text { maximize } w_{\min } \\
& w_{\text {min }} \leq x_{i} w_{i}+\left(1-x_{i}\right) \quad \forall i \in I \\
& \sum_{j \in J} y_{i j} \times \text { Demand }_{j} \leq \operatorname{cap}_{i} \times x_{i} \quad \forall i \in I \\
& \sum_{i \in I} y_{i j}=1 \quad \forall j \in J \\
& d_{i}^{\text {health }} \times x_{i} \leq \text { DistHealth } \forall i \in I \\
& d_{i}^{\text {road }} \times x_{i} \leq \text { DistRoad } \forall i \in I \\
& \frac{\sum_{j \in J} y_{i j} \times \text { Demand }_{j}}{\operatorname{cap}_{i}} \geq \beta x_{i} \quad \forall i \in I \\
& \frac{\sum_{j \in J} y_{i j} \times \text { Demand }_{j}}{\operatorname{cap}_{i}}-\frac{\sum_{j \in J} y_{k j} \times \text { Demand }_{j}}{\operatorname{cap}_{k}} \\
& \leq \alpha+\left(1-x_{i}\right)+\left(1-x_{k}\right) \quad \forall i \in I, k \in I, i \neq k \\
& y_{\text {distSorted }(1, j), j}=x_{\text {distSorted }(1, j)} \quad \forall j \in J \\
& y_{\text {distSorted }(i, j), j} \geq x_{\text {distSorted }(i, j)}-\sum_{k=1}^{i-1} x_{\operatorname{distSorted}(k, j)} \quad \forall j \in J, i=2 \ldots|I|
\end{aligned}
$$

$$
x_{i} \in\{0,1\} \quad \forall i \in I
$$

$y_{i j} \in\{0,1\} \quad \forall i \in I, j \in J$

(1) is the objective function that maximizes the minimum weight of open shelter areas. (2) bounds $w_{\min }$ above so that the maximum value that this variable can take is in fact the minimum weight of open shelter areas. Constraint (3) ensures that the total number of people in each shelter area does not exceed its capacity and no one gets assigned to a shelter area unless it is open. Constraint (4) states that each district needs to be assigned to a shelter area. Constraints (5) and (6) apply the threshold distance rule of main roads and health institutions. If there does not exist a main road and a health institution within the specified threshold distance, then the shelter area is not eligible to operate. Otherwise, it can either be operating or closed. Alternatively, the user can preprocess the data and identify the ineligible candidate shelter sites, remove them from the candidate set and omit constraints (5) and (6). Constraints (7) and (8) control the utilization of operating shelter areas. Constraint (7) states that if a shelter area is open, then the utilization of that shelter area needs to exceed the pre-determined threshold value. Otherwise, it becomes redundant. Constraint (8) states that for any pair of open shelter areas, the difference of their utilization needs to be less than the respective threshold value. Constraints (9a) and (9b) ensure that each district is assigned to the closest open shelter area, as suggested by Church and Roberts (1983). A shelter area is either open or closed. Therefore, constraint (10a) states that the decision variable regarding the status of each shelter area is binary. Moreover, since we do not allow partial assignments, the decision variable regarding the status of each district - shelter area assignment is binary as well, and is stated by constraint (10b).
The mathematical model above is developed for planning purposes. As one might see in the formulation, the model contains a variety of parameters. In an emergency situation, the decision maker may not be able to obtain the best values for each parameter. However, before a disaster occurs, it is possible to create many scenarios by varying these parameters, and obtain a plan for each scenario. The decision maker can then choose to execute the most suitable plan if and when a disaster hits.

To generate scenarios by varying the parameters, the decision makers can use various techniques. For example, for the dhealth parameter, which is the distance from a candidate shelter area to its closest health institution, the decision maker can identify 10 different routes and use their length randomly in each scenario. Taking a more advanced approach is possible as well. The decision maker can increase the number of distinct routes, use statistical tools to obtain a theoretical distribution and generate random variates. For $\alpha$ and $\beta$, the decision maker can use values from past occasions, or can take a more naïve approach by altering these parameters with fixed increments/decrements.

In an extreme case, some health institutions or some roads may be damaged, or in some cases they may be destroyed because of the disaster. In using the mathematical model for planning purposes, the decision maker can evaluate various what-if scenarios related to this issue. To do so, the decision maker can re-calculate the distance from each candidate shelter site to its closest main road and health institution, or remove a site from the candidate set and re-run the mathematical model.

Moreover, performing a sensitivity analysis on the above mentioned mathematical model can provide managerial insights to the decision makers. By observing the relationship between the input parameters and the objective value, decision makers can determine the most critical values for the parameters and by further scrutinizing each individual solution, they can identify the "critical" shelter areas (i.e. the facilities that are used in most of the solutions or the facilities that are highly utilized whenever they are in use).

This model has been developed for criteria of Turkish Red Crescent. However it can be easily modified for other organizations. Moreover, in this study the threshold distances were designed for main roads or hospitals. However, instead of those two, the decision maker can easily to adapt these constraints to other facilities, such as ports, warehouses, airports, etc.

\section{Computational studies}

\subsection{The case of Kartal, Istanbul, Turkey}

For computational studies, we use sample data based on the Kartal district of Istanbul, Turkey. The locations of candidate shelter areas, their weights and capacities are obtained from Unal (2010). Weights of candidate shelter areas differ from 0.674 to 0.982 with an average of 0.827 and a standard deviation of 0.097. Similarly, capacities of the candidate sites differ from 24,000 square meters to 150,000 square meters and the average capacity is 49,309 square meters. The district data are obtained from Google Maps and the population data are obtained from the Turkish Statistical Institute.

Kartal is one of the 39 districts of Istanbul. Its surface area is 38.54 square kilometers and it is the thirteenth most populous district of Istanbul, with approximately 425,000 inhabitants. In Kartal, there are 20 sub-districts and 25 potential shelter area locations. As in Unal (2010), we assumed that approximately 12.5 percent of the population would need to stay in shelter areas after an earthquake. Keeping the percentage of the affected population constant for all sub-districts may not seem to be a reasonable approach when the study involves a large geography. However, for studies involved in relatively small geographies such as Kartal, we believe that keeping this percentage constant will not alter the results dramatically. 


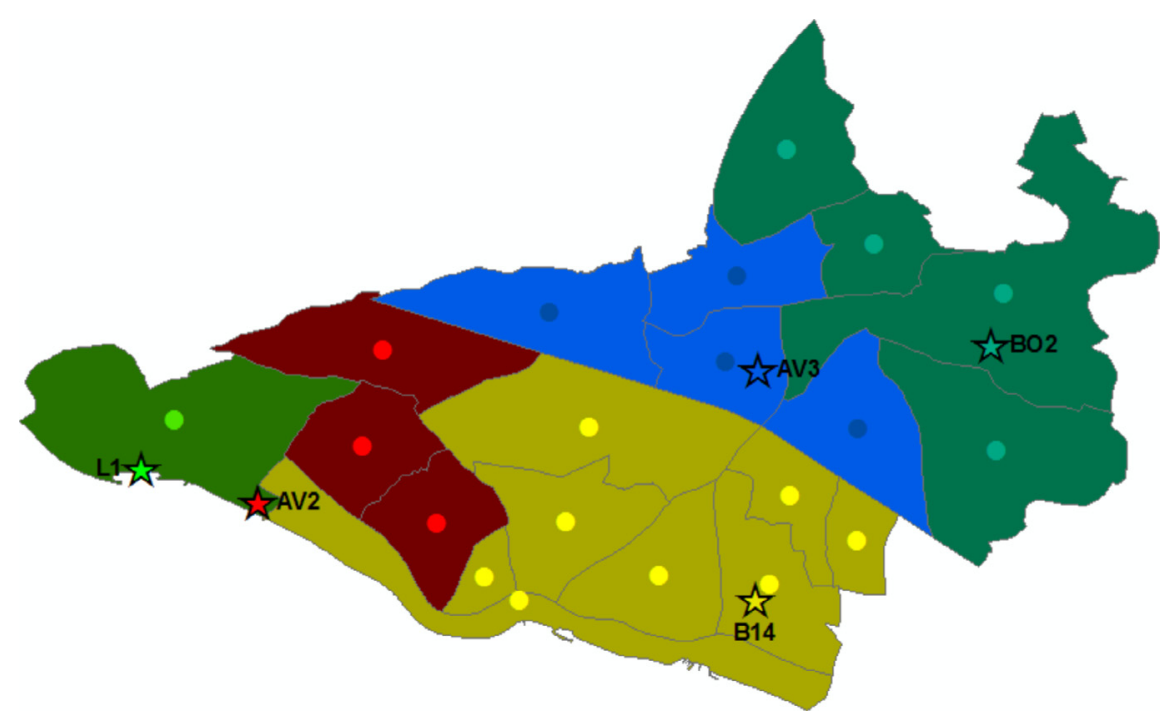

Fig. 1. Solution for the basic scenario result for the Kartal district.

The Turkish Red Crescent assigns 3.5 square meters of living space to each resident and 45 square meters for group sanitary and dining facilities. In our computations, we use these measures.

We obtain distance data from ESRI ArcGIS Desktop, a commercial geographical information system. Using Kartal's road network, candidate shelter area locations, health institutions, and main road junctions are pinned on the map. The centroid of each sub-district is chosen as the representation point. By using the Network Analyst extension of ESRI ArcGIS Desktop, we calculate the distances from shelter areas to sub-district centroids, health institutions, and main road junctions for the mathematical model.

We first generate a basic scenario where each shelter area is at least 60 percent utilized, their pairwise utilization difference is less than 20 percent, and there should be a hospital within 5 kilometers at most of each shelter area. Furthermore, for ease of procurement, main roads should be within 5 kilometers of all shelter areas. We solved the mathematical model using these parameters in less than 1 second. The results are schematized in Fig. 1.

In this map, stars represent the open shelter areas, solid circles represent district centroids and lines represent the assignments. There are five open shelter areas. The minimum of their weights is 0.827 and their average utilization is 0.863 . Also, the longest travel distance from a district to a shelter area is 3.8 kilometers, which is traversed by the residents of Ugurmumcu, the northmost sub-district.

Moreover, one can observe in the map that a portion of the population are assigned to the lower-right shelter area B14, which is in fact closer to shelter area AV2. A similar case can also be observed for shelter areas AV3 and BO2. Such cases are likely to occur when irregular-shaped sub-districts are present, which can be resolved by decreasing the level of aggregation. However, since the most disaggregated data available for our study was at the sub-district level, we conducted all of our computations at this level of aggregation.

To observe the behavior of the mathematical model with respect to problem parameters, we generate 3000 different scenarios by varying $\beta$ (threshold value for minimum utilization), $\alpha$ (threshold value for maximum pairwise utilization difference), and DistHealth and DistRoad (threshold distances from shelter areas to the nearest health institution and to the nearest main road). In our instances, $\beta$ varies from 0 to 0.9 with increments of 0.1 , and $\alpha$ varies from 1 to 0.1 with decrements of 0.1. For DistHealth we use 5, 4, 3, 2.5, 2, and 1.5 kilometers and for DistRoad we use 5, 4, 3, 2.5, and 2 kilometers. We use a PC with an Intel Core2Duo T6400 (2.0 gigahertz) processor, 4 gigabytes RAM and Gurobi 4.5.2 to compute the optimal solutions. Given the dataset, all instances are solved in less than a second.

We observe that as we decrease DistHealth and DistRoad values, the maximum of the minimum weight of open shelter areas decreases, and eventually the problem becomes infeasible. As DistHealth and DistRoad became smaller, the number of eligible shelter areas decreases. Because of this, the model is forced to select shelter areas with smaller weights, and eventually the objective value decreases. The tables below (Tables 4a, 4b, and 4c) show the objective values with different $\beta$ and $\alpha$ values, with DistHealth and DistRoad 5; DistRoad 5 and DistHealth 1.5; and DistRoad 2 and DistHealth 5 respectively.

From Table $4 \mathrm{a}$, we observe that the value of objective function is 0.85 for $\beta$ smaller than 0.7 and for $\alpha$ greater than 0.3 and after inspecting each solution individually; we observe that the solution of the mathematical model is different for each instance. However, the solution that is obtained when $\beta$ is equal to 0.6 and $\alpha$ is equal

Table 4a

The objective value when DistHealth $=$ DistRoad $=5$.

\begin{tabular}{lllllllllll}
\hline$\beta \backslash \alpha$ & 1 & 0.9 & 0.8 & 0.7 & 0.6 & 0.5 & 0.4 & 0.3 & 0.2 & 0.1 \\
\hline 0 & 0.85 & 0.85 & 0.85 & 0.85 & 0.85 & 0.85 & 0.85 & 0.85 & 0.827 & 0.827 \\
0.1 & 0.85 & 0.85 & 0.85 & 0.85 & 0.85 & 0.85 & 0.85 & 0.85 & 0.827 & 0.827 \\
0.2 & 0.85 & 0.85 & 0.85 & 0.85 & 0.85 & 0.85 & 0.85 & 0.85 & 0.827 & 0.827 \\
0.3 & 0.85 & 0.85 & 0.85 & 0.85 & 0.85 & 0.85 & 0.85 & 0.85 & 0.827 & 0.827 \\
0.4 & 0.85 & 0.85 & 0.85 & 0.85 & 0.85 & 0.85 & 0.85 & 0.85 & 0.827 & 0.827 \\
0.5 & 0.85 & 0.85 & 0.85 & 0.85 & 0.85 & 0.85 & 0.85 & 0.85 & 0.827 & 0.827 \\
0.6 & 0.85 & 0.85 & 0.85 & 0.85 & 0.85 & 0.85 & 0.85 & 0.85 & 0.827 & 0.827 \\
0.7 & 0.827 & 0.827 & 0.827 & 0.827 & 0.827 & 0.827 & 0.827 & 0.827 & 0.827 & 0.827 \\
0.8 & 0.827 & 0.827 & 0.827 & 0.827 & 0.827 & 0.827 & 0.827 & 0.827 & 0.827 & 0.827 \\
0.9 & 0.739 & 0.739 & 0.739 & 0.739 & 0.739 & 0.739 & 0.739 & 0.739 & 0.739 & 0.739 \\
\hline
\end{tabular}


Table 4b

The objective value when DistHealth $=1.5$ and DistRoad $=5$.

\begin{tabular}{lllllllllll}
\hline$\beta \backslash \alpha$ & 1 & 0.9 & 0.8 & 0.7 & 0.6 & 0.5 & 0.4 & 0.3 & 0.2 & 0.1 \\
\hline 0 & 0.739 & 0.739 & 0.739 & 0.739 & 0.739 & 0.739 & 0.739 & INF & INF & INF \\
0.1 & 0.739 & 0.739 & 0.739 & 0.739 & 0.739 & 0.739 & 0.739 & INF & INF & INF \\
0.2 & 0.739 & 0.739 & 0.739 & 0.739 & 0.739 & 0.739 & 0.739 & INF & INF & INF \\
0.3 & 0.739 & 0.739 & 0.739 & 0.739 & 0.739 & 0.739 & 0.739 & INF & INF & INF \\
0.4 & 0.739 & 0.739 & 0.739 & 0.739 & 0.739 & 0.739 & 0.739 & INF & INF & INF \\
0.5 & 0.739 & 0.739 & 0.739 & 0.739 & 0.739 & 0.739 & 0.739 & INF & INF & INF \\
0.6 & 0.739 & 0.739 & 0.739 & 0.739 & 0.739 & 0.739 & 0.739 & INF & INF & INF \\
0.7 & INF & INF & INF & INF & INF & INF & INF & INF & INF & INF \\
0.8 & INF & INF & INF & INF & INF & INF & INF & INF & INF & INF \\
0.9 & INF & INF & INF & INF & INF & INF & INF & INF & INF & INF \\
\hline
\end{tabular}

Table 4c

The objective value when DistHealth $=5$ and DistRoad $=2$.

\begin{tabular}{lllllllllll}
\hline$\beta \backslash \alpha$ & 1 & 0.9 & 0.8 & 0.7 & 0.6 & 0.5 & 0.4 & 0.3 & 0.2 & 0.1 \\
\hline 0 & 0.827 & 0.827 & 0.827 & 0.827 & 0.827 & 0.827 & 0.827 & 0.827 & 0.739 & 0.739 \\
0.1 & 0.827 & 0.827 & 0.827 & 0.827 & 0.827 & 0.827 & 0.827 & 0.827 & 0.739 & 0.739 \\
0.2 & 0.827 & 0.827 & 0.827 & 0.827 & 0.827 & 0.827 & 0.827 & 0.827 & 0.739 & 0.739 \\
0.3 & 0.827 & 0.827 & 0.827 & 0.827 & 0.827 & 0.827 & 0.827 & 0.827 & 0.739 & 0.739 \\
0.4 & 0.827 & 0.827 & 0.827 & 0.827 & 0.827 & 0.827 & 0.827 & 0.827 & 0.739 & 0.739 \\
0.5 & 0.827 & 0.827 & 0.827 & 0.827 & 0.827 & 0.827 & 0.827 & 0.827 & 0.739 & 0.739 \\
0.6 & 0.827 & 0.827 & 0.827 & 0.827 & 0.827 & 0.827 & 0.827 & 0.827 & 0.739 & 0.739 \\
0.7 & 0.739 & 0.739 & 0.739 & 0.739 & 0.739 & 0.739 & 0.739 & 0.739 & 0.739 & 0.739 \\
0.8 & 0.739 & 0.739 & 0.739 & 0.739 & 0.739 & 0.739 & 0.739 & 0.739 & 0.739 & 0.739 \\
0.9 & 0.739 & 0.739 & 0.739 & 0.739 & 0.739 & 0.739 & 0.739 & 0.739 & 0.739 & 0.739 \\
\hline
\end{tabular}

to 0.3 is feasible for all other instances with an objective value 0.85 . Thus, we can say that there are plenty of alternative optimal solutions. Similarly, for the cases where the value of the objective function is 0.827 , a similar case occurs with two different solutions.

However, when $\beta$ is 0.9 , the utilization difference of the shelter areas can be at most 0.1 . Thus, for all choices of $\alpha$, the mathematical model has the same feasible set and therefore reports the same solution. This situation implies that if the $\beta$ is chosen beforehand, $\alpha$ can be at most $1-\beta$. If $\alpha$ is chosen to be greater $1-\beta$, then the feasible set of the problem will be the identical to the feasible set of the problem where $\alpha$ is equal to $1-\beta$. Similarly, if $\alpha$ is chosen first, then $\beta$ needs to be at most $1-\alpha$. In general, we can say it is logical to choose $\alpha$ and $\beta$ in a fashion that their sum should be at most 1 .

Also, when we increase $\beta$ and decrease $\alpha$ while keeping DistHealth and DistRoad constant, the average utilization of open shelter areas increases. This increase is expected because as $\beta$ (the minimum utilization threshold) increases the minimum utilization of open shelter areas increases. Table 5 shows the average of the average utilization over 30 different DistHealth and DistRoad combinations with different $\beta$ and $\alpha$ values.

Moreover, as the objective value changes, the set of open shelter areas are not identical, but they are not totally different. For example, consider the case where DistHealth is 5 and DistRoad is 2. Among different values of $\beta$ and $\alpha$, there are two different objective values, 0.739 and 0.827 . In all cases where the objective value is the same, the set of open shelter areas are the same. However, when the set of open shelter areas of these two cases are inspected, it can be seen that the two sets are not identical and their intersection is not empty. Given two solutions, if the set of open shelter areas is identical, then the assignment between districts and open shelter areas is identical as well. On the contrary, if two sets are not identical, then the assignments are not identical.

As evident from Table 5 , when $\beta$ is 0.7 , the average of the average utilization does not change until $\alpha$ is decreased to 0.2 . A similar case occurs when $\beta$ is 0.8 and $\alpha$ is between 1.0 and 0.2 and when $\beta$ is 0.9 . This means that in those cases, regardless of DistHealth and DistRoad, the mathematical model yields the same optimal solution.

Similarly, as we increase $\beta$ and decrease $\alpha$ while keeping DistHealth and DistRoad constant, the number of open shelter areas decreases. This is expected because as the minimum utilization threshold increases the model tries to utilize the open shelter areas more and more and therefore it opens fewer shelter areas. Table 6 shows the maximum number of open shelter areas over 30 different DistHealth and DistRoad combinations with different $\beta$ and $\alpha$ values.

In Table 6 , we observe that when $\beta$ is 0.9 , at most two shelter areas are opened in the solution. When the same data for the minimum

Table 5

The average of the average utilization.

\begin{tabular}{lllllllllll}
\hline$\beta \backslash \alpha$ & 1.0 & 0.9 & 0.8 & 0.7 & 0.6 & 0.5 & 0.4 & 0.3 & 0.2 & 0.1 \\
\hline 0.0 & 0.671 & 0.687 & 0.692 & 0.720 & 0.779 & 0.779 & 0.787 & 0.797 & 0.875 & 0.875 \\
0.1 & 0.688 & 0.691 & 0.716 & 0.724 & 0.755 & 0.769 & 0.792 & 0.798 & 0.875 & 0.875 \\
0.2 & 0.711 & 0.711 & 0.717 & 0.725 & 0.764 & 0.771 & 0.785 & 0.785 & 0.877 & 0.877 \\
0.3 & 0.717 & 0.713 & 0.697 & 0.713 & 0.756 & 0.763 & 0.793 & 0.797 & 0.877 & 0.877 \\
0.4 & 0.761 & 0.758 & 0.762 & 0.762 & 0.762 & 0.764 & 0.797 & 0.798 & 0.875 & 0.875 \\
0.5 & 0.782 & 0.783 & 0.777 & 0.777 & 0.783 & 0.783 & 0.808 & 0.808 & 0.872 & 0.885 \\
0.6 & 0.804 & 0.815 & 0.814 & 0.814 & 0.814 & 0.814 & 0.814 & 0.823 & 0.899 & 0.918 \\
0.7 & 0.880 & 0.880 & 0.880 & 0.880 & 0.880 & 0.880 & 0.880 & 0.880 & 0.881 & 0.918 \\
0.8 & 0.904 & 0.904 & 0.904 & 0.904 & 0.904 & 0.904 & 0.904 & 0.904 & 0.904 & 0.933 \\
0.9 & 0.971 & 0.971 & 0.971 & 0.971 & 0.971 & 0.971 & 0.971 & 0.971 & 0.971 & 0.971 \\
\hline
\end{tabular}


Table 6

The maximum number of shelter areas opened.

\begin{tabular}{lllllllllll}
\hline$\beta \backslash \alpha$ & 1 & 0.9 & 0.8 & 0.7 & 0.6 & 0.5 & 0.4 & 0.3 & 0.2 & 0.1 \\
\hline 0 & 8 & 8 & 6 & 6 & 6 & 6 & 6 & 6 & 5 & 5 \\
0.1 & 7 & 7 & 7 & 6 & 6 & 6 & 6 & 6 & 5 & 5 \\
0.2 & 6 & 6 & 6 & 6 & 6 & 6 & 6 & 6 & 5 & 5 \\
0.3 & 5 & 5 & 5 & 5 & 5 & 5 & 5 & 4 & 4 & 4 \\
0.4 & 5 & 5 & 5 & 5 & 5 & 5 & 5 & 4 & 4 & 4 \\
0.5 & 5 & 5 & 5 & 5 & 5 & 5 & 5 & 4 & 4 & 4 \\
0.6 & 5 & 5 & 4 & 4 & 4 & 4 & 4 & 4 & 4 & 4 \\
0.7 & 5 & 5 & 4 & 4 & 4 & 4 & 4 & 4 & 4 & 4 \\
0.8 & 5 & 5 & 4 & 4 & 4 & 4 & 4 & 4 & 4 & 4 \\
0.9 & 2 & 2 & 2 & 2 & 2 & 2 & 2 & 2 & 2 & 2 \\
\hline
\end{tabular}

Table 7

Number of infeasibility cases for each $\alpha, \beta$ pair.

\begin{tabular}{lrrrrrrrrrr}
\hline$\beta \backslash \alpha$ & 1 & 0.9 & 0.8 & 0.7 & 0.6 & 0.5 & 0.4 & 0.3 & 0.2 & 0.1 \\
\hline 0 & 2 & 2 & 2 & 2 & 2 & 2 & 2 & 7 & 8 & 10 \\
0.1 & 2 & 2 & 2 & 2 & 2 & 2 & 2 & 7 & 8 & 10 \\
0.2 & 2 & 2 & 2 & 2 & 2 & 2 & 2 & 7 & 8 & 10 \\
0.3 & 2 & 2 & 2 & 2 & 2 & 2 & 2 & 7 & 8 & 10 \\
0.4 & 2 & 2 & 2 & 2 & 2 & 2 & 2 & 7 & 8 & 10 \\
0.5 & 2 & 2 & 2 & 2 & 2 & 2 & 2 & 8 & 8 & 11 \\
0.6 & 2 & 2 & 2 & 2 & 2 & 2 & 2 & 8 & 8 & 13 \\
0.7 & 8 & 8 & 8 & 8 & 8 & 8 & 8 & 8 & 8 & 13 \\
0.8 & 10 & 10 & 10 & 10 & 10 & 10 & 10 & 10 & 10 & 13 \\
0.9 & 15 & 15 & 15 & 15 & 15 & 15 & 15 & 15 & 15 & 15 \\
\hline
\end{tabular}

number of shelter areas are inspected, we observe that the minimum is 2 for all cases when $\beta$ is 0.9 . This implies that when $\beta$ is 0.9 , regardless of DistHealth, DistRoad, and $\alpha$, the optimal solution is the same.

We also measure the number of infeasibilities in each $\alpha, \beta$ pair among 30 different combinations of DistHealth and DistRoad. As we increase $\beta$ and decrease $\alpha$, the number of infeasible cases increases. Table 7 shows the number of infeasible cases for each $\alpha, \beta$ pair.

When DistHealth is 2 or 1.5 and DistRoad is 2 , all instances return infeasible because there are not enough eligible shelter areas to house all the population. As evident from Table 7, when $\beta$ is less than or equal to 0.6 and $\alpha$ is greater than or equal to 0.4 , the only infeasible cases are the ones noted above. However, as we narrow the feasible set by increasing $\beta$ and decreasing $\alpha$, depending on the values of DistHealth and DistRoad, more infeasible cases occur.

(a)

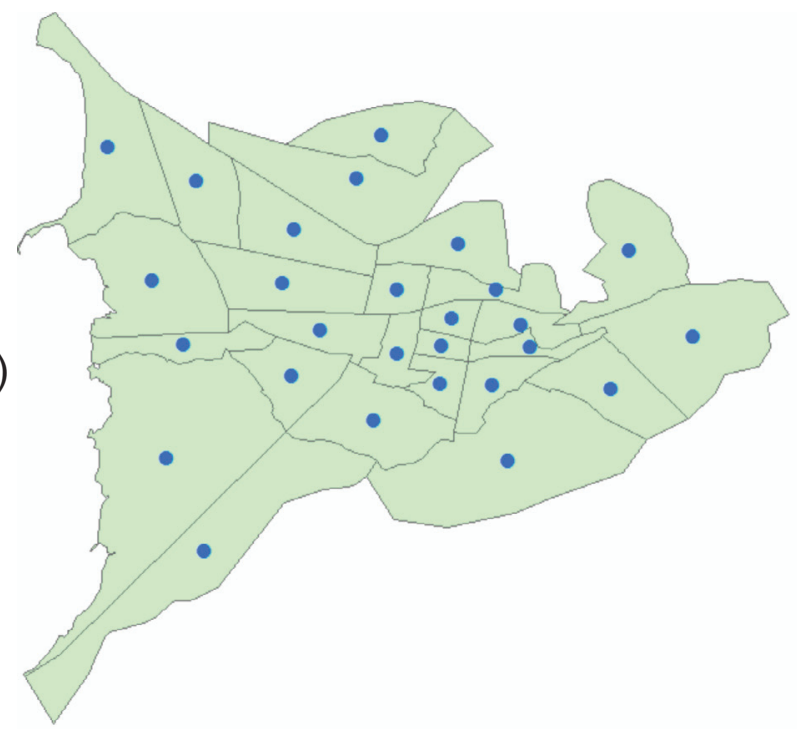

In all those 3000 instances, we assume that 12.5 percent of the population will be in need of temporary shelters. In addition to those instances, we generate 20 additional instances to observe the effect of the percentage of affected population on the value of the objective function. In those 21 instances, the values of $\beta, \alpha$, DistHealth and DistRoad are equal to the ones that we use in the base case scenario and the percentage of affected population changes from 10 to 20, with increments of 0.5 . From Table 8 , it can be observed that as the percentage of affected population increases, the value of the objective function decreases. The solution of each instance is different, even for the ones with the same objective value. However, in each solution that returned the same objective value, the shelter area with the minimum weight is the same. The objective value of each solution can be found in Table 8.

\subsection{Case study on Van, Turkey}

On October 23rd, 2011, an earthquake with a magnitude of 7.2 on the Richter scale hit the province of Van, Turkey. According to the Turkish Red Crescent's report, hundreds of thousands people were affected in Van and other surrounding cities. Along with other relief operations, the Turkish Red Crescent established four shelter sites in Van in order to provide living area to those in need.

In order to compare the proposed model with the Turkish Red Crescent's methodology, we perform a case study on the Van earthquake. To do so, in addition to the four shelter areas that the Turkish Red Crescent had determined, we identify 40 additional candidate shelter locations. The candidate locations are empty lots and

Table 8

The value of the objective function with different values of percent affected.

\begin{tabular}{llll}
\hline Percent affected & Objective & Percent affected & Objective \\
\hline 10 & 0.85 & 15.5 & 0.803 \\
10.5 & 0.85 & 16 & 0.801 \\
11 & 0.847 & 16.5 & 0.801 \\
11.5 & 0.847 & 17 & 0.801 \\
12 & 0.847 & 17.5 & 0.801 \\
12.5 & 0.827 & 18 & 0.801 \\
13 & 0.827 & 18.5 & 0.801 \\
13.5 & 0.809 & 19 & 0.801 \\
14 & 0.809 & 19.5 & 0.795 \\
14.5 & 0.803 & 20 & 0.739 \\
15 & 0.803 & & \\
\hline
\end{tabular}

(b)

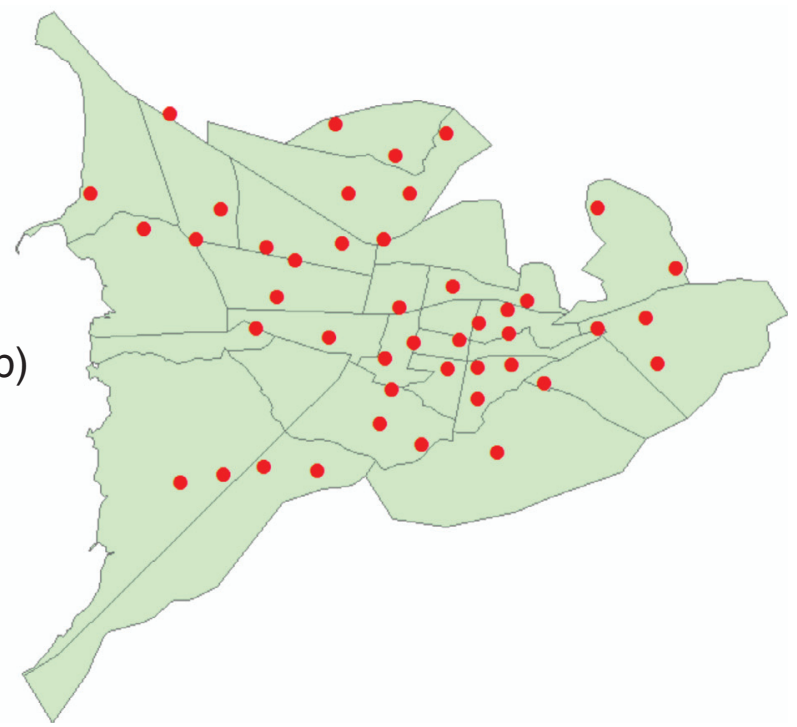

Fig. 2. (a) Centroids of Van's 27 districts. (b) Locations of 44 candidate shelter areas. 


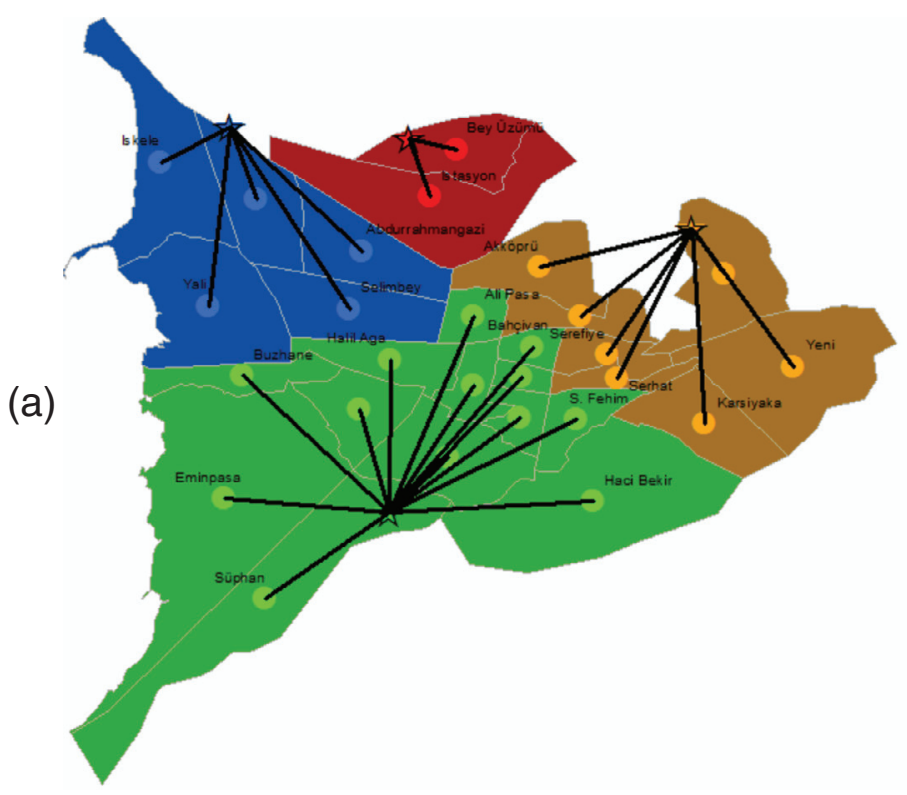

(b)

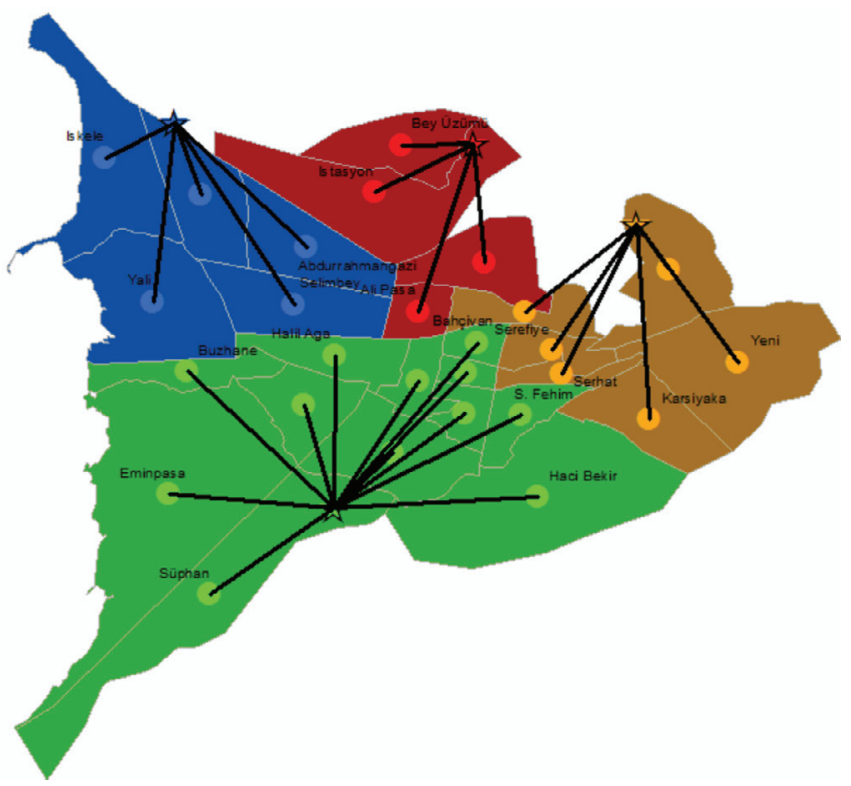

Fig. 3. (a) Turkish Red Crescent's solution. (b) Our solution.

Table 9

Comparison of Turkish Red Crescent's solution and our solution.

\begin{tabular}{lrr}
\hline & TRC's solution & Our solution \\
\hline The maximum distance $(\mathrm{m})$ & 5486.645 & 5486.645 \\
The minimum utilization & 0.095 & 0.277 \\
The maximum utilization & 0.767 & 0.967 \\
The average utilizaton & 0.375 & 0.569 \\
Total distance $(\mathrm{m})$ & 110607.517 & 107317.701 \\
\hline
\end{tabular}

schoolyards. For schoolyards, we take the capacity as 20,000 square meters on average and for the lots we take the capacity as 100,000 square meters on average. As in the Kartal case, we choose districts as the population areas. In the center of Van, there are 27 districts. The map of districts and candidate locations can be found in Fig. $2 \mathrm{a}$ and $\mathrm{b}$ respectively.

After determining the points representing the population candidate shelter area locations, we use our proposed model. As in the Kartal case, we calculate all necessary distances using ESRI ArcGIS Desktop and use 5 kilometers for the DistHealth and DistRoad parameters. For $\alpha$ and $\beta$, we use 1 and 0.2 , respectively. As the attributes of the candidate locations are not available to us, we assume the weight values of the candidate locations are equal to 0.5 . Moreover, in order to obtain a solution that is comparable to the one that the Turkish Red Crescent applied, we add a constraint in order to limit the number of open shelter areas to 4 . The map of the Turkish Red Crescent's solution and our solution can be found in Fig. 3a and b respectively. Also, the comparison of the two solutions can be found in Table 9.

As one can interpret from the two maps, the solutions are quite similar except the top-center shelter site. Our model decided to open a different shelter site in that zone and the population-shelter site assignment changed according to the nearest neighbor constraints. The comparison of the two solutions can be found in Table 9. One can see from the table that the difference between maximum and minimum utilizations does not differ significantly and the average utilization is to the midpoint of the range in both cases. Moreover, the maximum distance traversed by a person in both solutions is the same. However, it can be seen from the table that our solution is slightly better in terms of total distance travelled. Therefore, we can conclude that the Turkish Red Crescent's solution is already good enough, however, our methodology is able to find a slightly better one.

\section{Conclusion and future work}

In this study, we address the problem of locating temporary shelter areas after a disaster. We review the relevant literature and develop a mathematical model to select the best shelter area locations from a set of criteria. The model determines shelter area locations and matches population areas (districts) with the nearest open shelter while taking shelter area utilizations into account. We test the model using the data for Kartal, a district of Istanbul.

By varying the problem parameters, we test our model on 3000 different instances using the Kartal data. After inspecting the outputs, we observe that when the threshold distances to health institutions and main roads decrease, the objective value also decreases. Also, as the value of $\beta$ increases and the value of $\alpha$ decreases, the average utilization of open shelter areas increases and the number of open shelter areas decreases. Lastly, increasing the value of $\beta$ and decreasing the value of $\alpha$ result in an increase in the number of infeasibility cases over different DistHealth and DistRoad values. The model returns a solution for the Kartal case in less than 1 second.

We also implement our model with real data of the Van province in Turkey and compare our solution to the Turkish Red Crescent's implementation for the 2011 Van earthquake. Provided that the Turkish Red Crescent already has a good implementation, our solution is slightly better.

We mentioned earlier that the purpose of this mathematical model is to assist the planning stage of a possible disaster recovery process. Therefore, since an exact solution can be obtained in less than a second, we can assume that the decision maker has enough time to run the model for sufficiently large number of scenarios. Thus, development of a heuristic algorithm for this problem is not necessary at this stage.

Our model considers a deterministic case where the number of affected people is known. However, in the aftermath of a disaster, such data is usually not immediately available to decision makers. To compensate for this drawback, the mathematical model can be extended in a robust optimization model to consider different scenarios. 


\section{Acknowledgments}

We sincerely thank the three anonymous referees for contributing to the improvement of our paper. The corresponding author gratefully acknowledges support from the Turkish Academy of Sciences.

\section{References}

Ablanedo-Rosas, J. H., Gao, H., Alidaee, B., \& Teng, W. (2009). Allocation of emergency and recovery centres in Hidalgo, Mexico. International Journal of Services Sciences, 2(2), 206-218.

Alçada-Almeida, L., Tralhão, L., Santos, L., \& Coutinho-Rodrigues, J. (2009). A multiobjective approach to locate emergency shelters and identify evacuation routes in urban areas. Geographical Analysis, 41(1), 9-29.

Al Jazeera Turk (2013, November 28). Earthquakes with mass casualties in Turkey (in Turkish). Accessed 17 November 2014. http://www.aljazeera.com.tr/ dosya/turkiyede-buyuk-depremler

Anping, P. (2010). The applications of maximal covering model in typhoon emergency shelter location problem. In Industrial engineering and engineering management (IEEM), 2010 IEEE international conference on (pp. 1727-1731) IEEE.

Aslanzadeh, M., Rostami, E. A., \& Kardar, L. (2009). Logistics management and SCM in disasters. In R. Z. Farahani, N. Asgari, \& H. Davarzani (Eds.), Supply chain and logistics in national, international and governmental environment: Concepts and models (pp. 221-252). Berlin: Springer-Verlag.

Balcik, B., \& Beamon, B. M. (2008). Facility location in humanitarian relief. International Journal of Logistics, 11(2), 101-121.

Campbell, A. M., \& Jones, P. C. (2011). Prepositioning supplies in preparation for disasters. European Journal of Operational Research, 209(2), 156-165.

Chang, M. S., Tseng, Y. L., \& Chen, J. W. (2007). A scenario planning approach for the flood emergency logistics preparation problem under uncertainty. Transportation Research Part E: Logistics and Transportation Review, 43(6), 737-754.

Church, R. L., \& Roberts, K. L. (1983). Generalized coverage models and public facility location. In Papers of the regional science association: vo. 53, No. 1 (pp. 117-135) Springer-Verlag.

Coutinho-Rodrigues, J., Tralhão, L., \& Alçada-Almeida, L. (2012). Solving a locationrouting problem with a multiobjective approach: The design of urban evacuation plans. Journal of Transport Geography, 22, 206-218.

Dalal, J., Mohapatra, P. K., \& Mitra, G. C. (2007). Locating cyclone shelters: A case. Disaster Prevention and Management, 16(2), 235-244.

Dekle, J., Lavieri, M. S., Martin, E., Emir-Farinas, H., \& Francis, R. L. (2005). A Florida county locates disaster recovery centers. Interfaces, 35(2), 133-139.

Duran, S., Gutierrez, M. A., \& Keskinocak, P. (2011). Pre-positioning of emergency items for care international. Interfaces, 41(3), 223-237.

Görmez, N., Köksalan, M., \& Salman, F. S. (2011). Locating disaster response facilities in Istanbul. Journal of the Operational Research Society, 62(7), 1239-1252.

Gül, S. (2008). Post-disaster casualty logistics planning for Istanbul (MS thesis). Koç University.

Günneç, D. (2007). Network optimization problems for disaster mitigation: Network reliability, investment for infrastructure strengthening and emergency facility location (MS thesis). Koç University.

Hale, T., \& Moberg, C. R. (2005). Improving supply chain disaster preparedness: A decision process for secure site location. International Journal of Physical Distribution E' Logistics Management, 35(3), 195-207.

Han, Y., Guan, X., \& Shi, L. (2011). Optimization based method for supply location selection and routing in large-scale emergency material delivery. Automation Science and Engineering, IEEE Transactions on, 8(4), 683-693.

Huang, R., Kim, S., \& Menezes, M. B. (2010). Facility location for large-scale emergencies. Annals of Operations Research, 181(1), 271-286.

IFRC. (2012). What is a Disaster? Accessed 06 June 2012. <http://www.ifrc.org/ en/what-we-do/disaster-management/about-disasters/what-is-a-disaster/>

Jia, H., Ordóñez, F., \& Dessouky, M. M. (2007a). A modeling framework for facility location of medical services for large-scale emergencies. IIE Transactions, 39(1), 41-55.
Jia, H., Ordóñez, F., \& Dessouky, M. M. (2007b). Solution approaches for facility location of medical supplies for large-scale emergencies. Computers \& Industrial Engineering 52(2), 257-276

Kapucu, N., Lawther, W. C., \& Pattison, S. (2007). Logistics and staging areas in managing disasters and emergencies. Journal of Homeland Security and Emergency Management, 4(2).

Li, A. C., Nozick, L., Xu, N., \& Davidson, R. (2012). Shelter location and transportation planning under hurricane conditions. Transportation Research Part E: Logistics and Transportation Review, 48(4), 715-729.

Li, L., Jin, M. (2010). Sheltering Planning and Management for Natural Disasters. THCIT-2010 Conference \& Exhibition.

Li, L., Jin, M., \& Zhang, L. (2011). Sheltering network planning and management with a case in the Gulf Coast region. International Journal of Production Economics, 131(2), 431-440.

Liu, Q., Ruan, X., \& Shi, P. (2011). Selection of emergency shelter sites for seismic disasters in mountainous regions: Lessons from the 2008 Wenchuan Ms 8.0 Earthquake, China. Journal of Asian Earth Sciences, 40(4), 926-934.

Lu, C. C. (2013). Robust weighted vertex p-center model considering uncertain data: An application to emergency management. European Journal of Operational Research, 230(1), 113-121.

Lu, X. L., \& Yun-xian, H. (2009). Ant colony optimization for facility location for largescale emergencies. In Management and Service Science, 2009. MASS'09. International Conference on (pp. 1-4) IEEE.

Mete, H. O., \& Zabinsky, Z. B. (2010). Stochastic optimization of medical supply location and distribution in disaster management. International Journal of Production Economics, 126(1), 76-84.

Milliyet (2000, August 11-16). 175,000 people are still homeless (in Turkish). Accessed 6 June 2012. <http://www.milliyet.com.tr/ozel/depremdosyasi/index.html>

Murali, P., Ordóñez, F., \& Dessouky, M. M. (2012). Facility location under demand uncertainty: Response to a large-scale bio-terror attack. Socio-Economic Planning Sciences, 46(1), 78-87.

Özmen, B., Nurlu, M., \& Güler, H. (1997). Inspection of earthquake zones of Turkey with geographical information systems (in Turkish). Directorate of Disaster Operations, Department of Earthquake Research.

Özmen, B., Nurlu, M., Kuterdem, K., \& Temiz, A. (2005). Directorate of disaster operations, earthquake sympozium. (in Turkish). Kocaeli, Turkey: Grand Yükseliş Hotel.

Paul, J. A., \& Batta, R. (2008). Models for hospital location and capacity allocation for an area prone to natural disasters. International Journal of Operational Research, 3(5), 473-496.

Radikal (2000, March 16) Days are Passed in Tents (in Turkish). Accessed 06 June 2012 http://www.radikal.com.tr/2000/03/16/t/turkiye/01mev.shtml

Rath, S., \& Gutjahr, W. J. (2014). A math-heuristic for the warehouse location-routing problem in disaster relief. Computers E Operations Research, vol. 42, 25-39.

Rawls, C. G., \& Turnquist, M. A. (2010). Pre-positioning of emergency supplies for disaster response. Transportation Research Part B: Methodological, 44(4), 521-534.

Sphere Project (2011). Sphere Project Humanitarian Charter and Minimum Standards in Disaster Response.

Toro-Díaz, H., Mayorga, M. E., Chanta, S., \& McLay, L. A. (2013). Joint location and dispatching decisions for Emergency Medical Services. Computers \&' Industrial Engineering, 64(4), 917-928.

Turkish Red Crescent. (2013). Report on Humanitarian Operations in Van. Accessed 19 June 2013. <http://afetyonetimi.kizilay.org.tr/Operasyonlar.aspx?Id=4>

Unal, G. (2010). Acil Lojistik Yardım Operasyonlarında (Deprem Lojistiği) Kullanılacak Bir "Model Tabanlı Lojistik Karar Destek Sistemi" Geliștirilmesi (A mathematical model based decision support system for emergency operations) (Ph. D. dssertation). Turkish Military Academy Defense Sciences Institutes.

Verma, A., \& Gaukler, G. M. (2011). A stochastic optimization model for positioning disaster response facilities for large scale emergencies. In Network optimization (pp. 547-552). Heidelberg: Springer, Berlin.

Wang, D. W., \& Zhang, G. X. (2006). Model and algorithm for optimization of rescue center location of emergent catastrophe. Frontiers of Electrical and Electronic Engineering in China, 1(3), 265-268.

Yushimito, W. F., Jaller, M., \& Ukkusuri, S. (2012). A Voronoi-based heuristic algorithm for locating distribution centers in disasters. Networks and Spatial Economics, 12(1), 21-39. 